\title{
Enfermagem na assistência centrada ao paciente em unidade de terapia intensiva: contribuição de um display informativo
}

Nursing in patient-centered care in an intensive care unit: contribution of an informative display

Enfermería en atención centrada en el paciente en una unidad de atención intensiva: contribución de una pantalla informativa

\begin{abstract}
RESUMO
Objetivo: Analisar as metas traçadas através de um display informativo à beira leito, a partir da perspectiva da experiência do paciente e discutir as metas diárias descritas à luz do cuidado centrado no paciente. Métodos: Pesquisa qualitativa do tipo exploratória, descritiva e documental. Realizado de maio a julho de 2019, em uma Unidade de Terapia Intensiva cardíaca de um hospital público universitário do município do Rio de Janeiro. Os dados foram coletados através de um display informativo. Resultados: Foram analisados 30 displays, citado 88 metas pelos pacientes, organizadas em três dimensões. A meta mais mencionada pelos pacientes esteve relacionada à alta hospitalar, saúde e bem estar, relatada por $22(78,8 \%)$ vezes no display informativo à beira leito. Conclusão: 0 estudo concluiu que o display informativo à beira leito poderá contribuir como uma ferramenta facilitadora de comunicação de metas do paciente e estabelecer estratégias para oferta do cuidado centrado no paciente.

DESCRITORES: Assistência de Enfermagem; Assistência centrada no paciente. Comunicação em saúde. Segurança do paciente. Unidade de Terapia Intensiva.
\end{abstract}

\section{ABSTRACT}

Objective: To analyze the goals set through an informative display at the bedside, from the perspective of the patient's experience and discuss the daily goals described in the light of patient-centered care. Methods: Qualitative, exploratory, descriptive and documentary research. Held from May to July 2019, in a cardiac intensive care unit of a public university hospital in the city of Rio de Janeiro. The data were collected through an informative display. Results: 30 displays were analyzed. The goal most mentioned by patients was related to hospital discharge, health and well-being, reported $22(78.8 \%)$ times on the bedside information display. Conclusion: The study concluded that the informative display at the bedside can contribute as a facilitating tool for communicating patient goals and establishing strategies for offering patient-centered care.

DESCRIPTORS: Nursing Care. Patient-Centered Care. Health Communication. Patient Safety. Intensive Care Units.

\section{RESUMEN}

Objetivo: analizar los objetivos establecidos a través de una pantalla informativa junto a la cama, desde la perspectiva de la experiencia del paciente y discutir los objetivos diarios descritos a la luz de la atención centrada en el paciente. Métodos: Investigación cualitativa, exploratoria, descriptiva y documental. Se lleva a cabo de mayo a julio de 2019, en una unidad de cuidados intensivos cardíacos de un hospital universitario público en la ciudad de Río de Janeiro. Los datos fueron recolectados a través de una pantalla informativa. Resultados: se analizaron 30 pantallas. El objetivo más mencionado por los pacientes se relacionó con el alta hospitalaria, la salud y el bienestar, se informó 22 (78.8\%) veces en la pantalla de información junto a la cama. Conclusión: El estudio concluyó que la exhibición informativa al lado de la cama puede contribuir como una herramienta facilitadora para comunicar los objetivos del paciente y establecer estrategias para ofrecer atención centrada en el paciente.

DESCRIPTORES: Cuidado de Enfermera. Atención Dirigida al Paciente. Comunicación en Salud. Seguridad del Paciente. Unidades de Cuidados Intensivos.

RECEBIDO EM: 25/06/2020 APROVADO EM: 06/08/2020 


\section{Raquel Rodrigues França de Castro}

Enfermeira do Hospital Universitário Pedro Ernesto.RJ.

ORCID: 0000-0003-3093-5280

\section{Andrezza Serpa Franco}

Professora Assistente do Departamento Médico Cirúrgico da Universidade do Estado do Rio de Janeiro- UERJ - RJ. ORCID: 0000-0001-5008-1345

\section{Karla Biancha Silva de Andrade}

Professora Adjunto do Departamento Médico Cirúrgico da Universidade do Estado do Rio de Janeiro- UERJ - RJ. ORCID: 0000-0002-6216-484X

\section{Luana Ferreira de Almeida}

Professora Adjunto do Departamento Médico Cirúrgico da Universidade do Estado do Rio de Janeiro- UERJ - RJ. ORCID: 0000-0001-8433-4160

Ana Lúcia Cascardo Marins Professora Assistente do Departamento Médico Cirúrgico da Universidade do Estado do Rio de Janeiro- UERJ - RJ. ORCID: 0000-0002-8485-8308

\section{Roberto Carlos Lyra da Silva}

Professor Associado do Departamento de Enfermagem Fundamental da Universidade Federal do Estado do Rio de JaneiroUNIRIO - RJ.

ORCID: 0000-0001-9416-9525

\section{INTRODUÇÃO}

0 cuidado centrado no paciente abarca o desenvolvimento de práticas entre pacientes e profissional de saúde para diminuir prováveis assimetrias nas relações. "Centrar no paciente" significa envolvê-lo na terapêutica, apoiar a sua participação e de seus familiares mediante troca de informações e principalmente respeitar a liberdade do paciente de decidir entre as opções de cuidado, acolhendo suas deliberações e necessidades físicas e emocionais $^{(1)}$.

$O$ cuidado centrado no paciente faz parte de um dos objetivos de um plano de melhoria de saúde nos Estados Unidos, conforme estabelecido no relatório Crossing the qualit chasm: new health system for the 21 st century, do Institute of Medicine (IOM). No documento, o cuidado centrado no paciente é definido como "a prestação de cuidado de forma respeitosa, respondendo às necessidades, preferências e valores da pessoa assistida, com a garantia de que tais valores norteiem todas as decisões clínicas ${ }^{1}$.

Embora haja evidências que a prática centrada no paciente produza efeitos positivos em resultados clínicos, aplicar no conceito é um desafio, uma vez que trata-se de um modelo de atenção que se propõe a romper paradigmas remanescentes do modelo biomédico na tentativa de minimizar a fragmentação do cuidado ${ }^{(1)}$.

Os desafios parecem ser ainda maiores em Unidades de Terapia Intensiva (UTI) destinadas a pacientes graves, uma vez que, fatores relacionados à comunicação ineficaz e relacionamento entre os profissionais e o paciente alteram a percepção do paciente sobre a qualidade do cuidado prestado $^{(2,3)}$.

Nessa perspectiva, em maio de 2019, o uso de um display informativo beira leito, contendo as metas diárias, sob a ótica dos pacientes, foi implementado em 2 leitos aleatórios não consecutivos em uma unidade cardio intensiva cirurgica. Embora todos os dados do display informativo como, nome do paciente, prontuário, idade, data, cirurgia realizada e meta clínica tivessem sido coletados no decorrer do estudo, foi optado em analisar a característica da população (gênero, idade, tipo de cirurgia cardíaca) e a meta do paciente com a pergunta "o que é importante pra mim hoje?”. O display tem como objetivo, expor as necessidades diárias dos pacientes, durante a sua hospitalização, a fim de facilitar a comunicação segura entre equipe de saúde e o paciente.

A coparticipação do paciente durante a sua especialização é visto ainda, como em fator de redução dos custos de saúde e de maior eficiência nos cuidados, na fase diagnóstica e tratamento, constituindo um determinante importante da relação equipe de saúde e pacientes, tornando a comunicação transparente e mais efetiva ${ }^{(2)}$.

Nos dias atuais, a constatação dos benefícios do envolvimento do paciente e família no processo do cuidado tem transformado ações de informação e de educação dos mesmos e dos profissionais, em recomendações institucionais e normas no âmbito de políticas de saúde, favorecendo a comunicação efetiva entre profissional e paciente ${ }^{(4,5)}$.

Este estudo está relacionado com as metas estipuladas pelos pacientes sobre sua hospitalização no pós operatório de cirurgia cardíaca em um display informativo à beira leito para nortear a equipe de saúde no planejamento da assistência centrada no paciente.

Diante deste contexto o objetivo deste estudo foi analisar as metas traçadas por 
pacientes, através de um display informativo à beira leito de uma Unidade Cardiointensiva Cirurgica.

\section{MÉTODOS}

Trata-se de uma pesquisa qualitativa do tipo exploratória, descritiva e documental, realizada no período de maio a julho de 2019, em uma UTI cardíaca de 12 leitos de um hospital público universitário do município do Rio de Janeiro.

Os dados foram coletados através de 2 displays fixos, em leitos aleatórios e não consecutivos. Nome do paciente, idade, gênero biológico, tipo de cirurgia realizada, meta do paciente extraída através da pergunta: "O que é importan-

Figura 1: Display informativo para exemplificar o modelo "piloto" disposto no leito de pós operatório de cirurgia cardíaca.

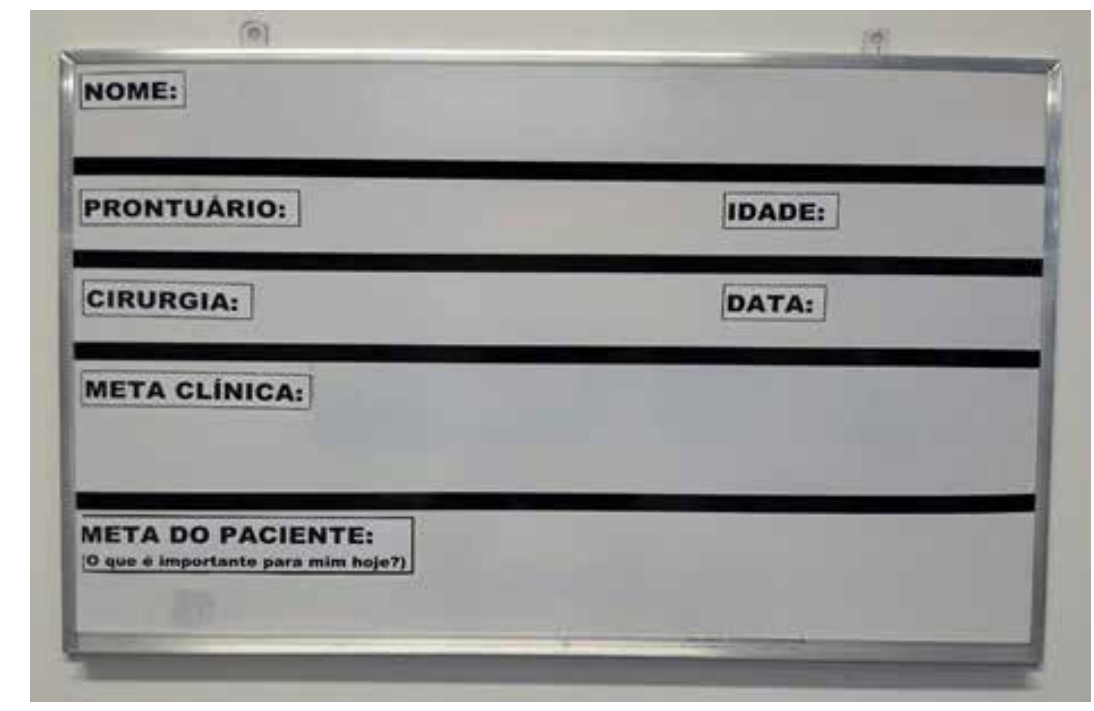

Fonte: Os autores, 2020

Tabela 1: Característica da população do estudo. Rio de Janeiro, RJ, Brasil, 2019.

$$
\text { Caracterização }
$$

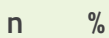

\section{Sexo Biológico}

Feminino

Masculino

Idade

20 a 59

$>60$

te para mim hoje?" e meta clinica estipulada pela equipe de saúde eram dados descritos no display. Para estudo foram coletados dados para descrever as características dos pacientes e as metas estamento com a equipe de saúde para o entendimento sobre significado de metas do paciente, experiência do paciente e a importância do referido registro. O preenchimento dos dados no display foi realizado pela equipe que assiste o paciente, de ordo com a escala diária de serviço.

Foram analisadas as fontes de informação dos pacientes: adultos, maiores de 18 anos, em pós-operatório de cirurgia cardíaca, e extubados com escada de sedação Ri-

Cirurgias 


\section{artigo}

Castro, R.R.F.; Franco, A.S.; Andrade, K.B.S.; Almeida, L.F.; Marins, A.L.; Lyra da Silva, R.C.

Enfermagem na assistência centrada ao paciente em unidade de terapia intensiva: contribuição de um display informativo

$\begin{array}{lcc}\text { RVM (Revascularização do miocárdio) } & 19 & 63 \\ \text { TVAO (Troca de valva aórtico) } & 4 & 13 \\ \text { RVM + TVAO (Revascularização do miocárdio + Troca de valva aórtico) } & 3 & 10 \\ \text { TVM (Troca valva mitral) } & 1 & 3 \\ \text { RVM + Ventriculoplastia (Revascularização do miocárdio) } & 1 & 3 \\ \text { Implante de Marca-passo } & 2 & 7 \\ \text { Fonte: Dados da pesquisa, 2020. } & & \end{array}$

Foram identificadas 88 metas, mencionadas pelos pacientes, organizadas em três dimensões: "Alta hospitalar, Saúde e Bem estar", "Necessidades básicas humanas" e "Religiosidade" (Quadro 1).

A meta mais mencionada pelos pacientes investigados esteve relacionada à alta hospitalar, saúde e bem estar, relatada por 22 (78,8\%), em seguida de necessidades básicas humanas por $14(15,4 \%)$ e religiosidade $3(5,8 \%)$ vezes no display informativo à beira leito.

\section{DISCUSSÃO}

Observou-se prevalência da cirurgia de Revascularização do Miocárdio (RVM), em pacientes do sexo biológico masculino e acima de 60 anos. A prevalência senil e masculina já é conhecida em outros estudos recentes, pode estar relacionada ao aumento da expectativa de vida e o crescente aumento da doença arterial coronariana, que pode evoluir para cirurgia cardíaca e em especial, revascularização do miocárdio ${ }^{(6,7)}$.
Em relação às categorias atribuídas às metas do paciente, a de maior prevalência no estudo esteve relacionada ao bem estar e alta hospitalar. Quanto menor o tempo de permanência do paciente no hospital, melhor parece ser seu prognóstico, sendo assim, o retorno precoce às atividades diárias e o convívio com seus familiares, pode contribuir para a sua reabilitação( ${ }^{(8)}$.

Em relação as metas dos pacientes estipuladas: "Sair bem da cirurgia"; "Que ocorra tudo bem no procedimento"; "Que a cirurgia ocorra bem", remeteu a um desejo de não complicação. As complicações pós-operatórias da cirurgia cardíaca são amplamente discutidas na literatura e sua incidência varia de acordo com fatores individuais, como idade, hábitos de vida, comorbidades associadas e situação clínica no momento da cirurgia, além de questões relacionadas ao processo cirúrgico, como tempo de duração da cirurgia, uso de circulação extracorpórea, agentes anestésicos utilizados e complicações no período transoperatório ${ }^{(9)}$.
Aliada a dimensão de bem estar e alta, a desospitalização precoce dos pacientes submetidos à cirurgia cardíaca vem sendo cada vez mais recomendada, com o propósito de diminuir o tempo de internação, complicações, gastos hospitalares, bem como oferecer ao paciente a possibilidade de retornar às suas atividades rotineiras e $o$ convívio com a família ${ }^{(10-13)}$.

No presente estudo, alguns fatores estressores foram identificados no display informativo como o distanciamento da família; privação do convívio com os filhos e netos; desconforto com presença de tubos necessários ao tratamento cirúrgico; persistência da dor física relacionada ao procedimento invasivo.

Neste sentido, o desejo da alta hospitalar e a busca de melhor bem estar confluem para as metas mencionadas como "Quero ficar com minha família"; "Ver os filhos"; "Encontrar os filhos"; "Ajudar minha esposa a cuidar dos meus filhos"; "Quero ficar com minha família"; "Curtir minha neta”; "Quero ver minha filha", "Acordar melhor"; "Parar de sentir dor"; "Andar"; "Ir embora", "Ver o sol". O entendimento de tais expressões possibilita a compreensão da necessidade de um cuidado integral, para além da necessidade física.

Outros fatores mencionados como desejos dos pacientes foram: "Retirar tubo"; "Retirar a máscara (VNI)"; "Retirar tudo que está em mim”; "Retirar borracha da

\section{Quadro1: Organização das dimensões atribuídas às metas estipuladas pelos pacientes. Rio de Janeiro, RJ, Brasil, 2019.}

\begin{tabular}{|c|c|}
\hline CATEGORIA ATRIBUÍDA & META ESTIPULADA PELO PACIENTE \\
\hline Alta hospitalar, Saúde e Bem estar & $\begin{array}{l}\text { "Ir para casa"; "Ir embora"; "Ter alta". "Ficar bom"; "Ter um bom atendimento"; "Ter uma vida normal"; } \\
\text { "Recuperar-me"; "Sair daqui saudável"; "Ficar melhor"; "Ser paciente"; "Ter saúde"; "Qualidade de } \\
\text { vida". } \\
\text { "Ver os filhos"; "Encontrar os filhos"; "Ajudar minha esposa a cuidar dos meus filhos"; "Quero ficar } \\
\text { com minha familia"; "Curtir minha neta"; "Quero ver minha filha". } \\
\text { "Retirar tubo"; "Retirar a máscara (VNII"; "Retirar tudo que está em mim"; "Retirar borracha da minha } \\
\text { barriga e do meu pênis". } \\
\text { "Sair bem da cirurgia"; "Que ocorra tudo bem no procedimento"; "Que a cirurgia ocorra bem". } \\
\text { "Parar de sentir dor"; "Tirar a dor"; "Sentir menos dor". } \\
\text { "Ver o sol". }\end{array}$ \\
\hline Necessidades básicas humanas & $\begin{array}{l}\text { "Fazer a cirurgia"; "Beber água"; "Operar"; "Acordar melhor"; "Colocar a prótese dentária (tartaruga)"; } \\
\text { "Realizar o procedimento"; "Caminhar"; "Sentar no sofá"; "Andar"; "Sair do leito e caminhar"; "Sentar } \\
\text { na poltrona"; "Realizar cirurgia"; "Melhorar respiração"; }\end{array}$ \\
\hline Religiosidade & "Quero paz"; "Quero Deus"; "Deus em primeiro lugar”. \\
\hline
\end{tabular}


minha barriga e do meu pênis"; "Ficar bom"; "Ter um bom atendimento"; "Ter uma vida normal"; "Recuperar-me"; "Sair daqui saudável"; "Ficar melhor"; "Ser paciente"; "Ter saúde"; "Qualidade de vida”.

Apesar de importantes e essenciais durante o período perioperatório, as tecnologias do cuidado, muitas vezes conhecidas como tecnologias invasivas (cateteres, tubos, drenos etc), demonstram dependência do paciente ao serviços de saúde, além de causar dor e desconforto. É necessário uma avaliação multidisciplinar para a retirada o quanto antes dos dispositivos, promovendo conforto, autonomia, bem estar , inclusive estabelecendo condicionantes para alta hospitalar ${ }^{(14,15)}$.

$\mathrm{Na}$ dimensão necessidades humanas básicas, os pacientes demonstraram o desejo e a importância de caminhar e sair do leito. A atividade de deambulação precoce requer uma análise, esforço e vontade da equipe multiprofissional. A mobilização precoce conceituada como terapia realizada na UTI em pacientes críticos, junto ao posicionamento adequado no leito, pode ser considerada como estimulação sensório-motora, prevenindo agravamentos de complicações neuromusculares causadas pelo imobilismo ${ }^{(16,17)}$.

Com a finalidade de cumprir o desejo e a necessidade física do paciente, é primordial que esse paciente se encontre em capacidade física adequada, com parâmetros hemodinâmicos estáveis e sem dispositivos que dificultam a deambulação como: drenos, cateter de pressão arterial invasiva, drogas vasoativa para controle pressão arterial, drogas cardiovasculares entre outros. A deambulação precoce não deve se centralizar apenas na prevenção de com- plicações e morbidades, como também na recuperação funcional do paciente ${ }^{(18-20)}$.

Ainda na dimensão relacionada às necessidades básicas como: "Colocar a prótese dentária", "Beber água”; "Acordar melhor" e "Melhorar respiração", remetem à teoria de Maslow de 1964, na qual as necessidades fisiológicas do indivíduo são as mais fortes, as mais básicas e primordiais para manutenção da saúde. Quando essas não são correspondidas, sobrevêm sentimentos de nervosismo, insônia, baixa autoestima, insegurança entre outros, podendo ser recompensados com sentimentos negativos. Oferecer uma assistência direcionada para atingimento das necessidades básicas acima das clínicas corrobora com a essência do cuidar em enfermagem.

A espiritualidade/religiosidade remete a relação com o transcendental, na busca de significados da vida. Entretanto, apesar de parecerem sinônimos, diferenciam-se pela sua abrangência; enquanto a religião se caracteriza como meio de buscar a espiritualidade através de uma instituição religiosa organizada, a espiritualidade não necessita estar atrelada a uma religião, sendo ela inerente ao indivíduo. A religiosidade também foi dimensionada como metas dos pacientes, identificada nas expressões: "Quero paz"; "Quero Deus"; "Deus em primeiro lugar".

A religiosidade favorece novo sentido à experiência do paciente sobre a doença, alterando a maneira como ele enxerga o problema, promovendo maior alívio da dor e da aflição. O bem-estar espiritual é considerado como um fator de proteção, relacionado a atitudes positivas de combate à enfermidade. $\mathrm{O}$ fortalecimento do bem-estar espiritual pode auxiliar na redução da angústia relacionada a doenças, bem como na promoção da saúde mental ${ }^{(21)}$.

A realização das metas estabelecidas pelos pacientes sobre o processo de cuidar não parece ser um ato isolado no pós-operatório de cirurgia cardíaca, nem todos os procedimentos técnicos realizados são resultados de um processo satisfatório. É preciso agregar a essas práticas, valores, deveres, direitos, sentimentos, atitudes e princípios. A enfermagem nesse contexto tem por função contribuir para o conforto e atribuir uma cultura de segurança, preparando-o para uma recuperação adequada.

Foi considerado como limitação neste estudo, o número reduzido de leitos com display informativo, restringindo o número de participantes, bem como em uma única unidade da instituição hospitalar.

\section{CONCLUSÃO}

O estudo concluiu que o display informativo à beira leito poderá contribuir como uma ferramenta facilitadora de comunicação das metas determinadas pelo paciente e a partir delas a equipe de saúde, nortear uma prática centrada no paciente.

Observou-se que durante a coleta de dados das através do display a valorização dos pacientes em expor seus desejos através de metas, é parte fundamental do processo de recuperação e pode contribuir para que o período de hospitalização esteja relacionado a uma boa experiência vivida, fortalecendo o elo entre paciente-profissional de saúde, configurando maior segurança e confiança nas relações.

\section{REFERÊNCIAS}

1. Paranhos Denise G. A. M., Albuquerque Aline, Garrafa Volnei. Vulnerabilidade do paciente idoso à luz do princípio do cuidado centrado no paciente. Saude soc. [Internet]. 2017 Dec [cited 2020 June 13] ; 26( 4 ): 932-942. Available from: http://www.scielo.br/scielo.php?script=sci_arttext\&pid=S0104-12902017000400932\&Ing=en. https://doi. org/10.1590/s0104-12902017170187
2. Smaradottir BF, Fensli RW. Evaluation of Technology Use in an Interdisciplinary Patient-centered Health Care Team. Studies in Health Tecnology and Informatics [internet].2019[cited 2018 jul 7];257: 388-392. Available from : 10.3233/978-1-61499951-5-388

3. Duarte SCM, Stipp MAC, Silva MM, Oliveira FT. Eventos adversos e segurança na assistência de enfermagem. Rev Bras 


\section{REFERÊNCIAS}

Enferm [internet].2015[cited 2018 dec 03]; 68 (1):144-54. Available from: http://www.scielo.br/pdf/reben/v68n1/00347167-reben-68-01-0144.pdf

4. Dordetto PR, Pinto GC, Rosa TCSC. Pacientes submetidos à cirurgia cardíaca: caracterização sócio demográfica, perfil clínico-epidemiológico e complicações. Rev Fac Ciên Méd Sorocaba[internet] .2016[cited 2018 marc 7];18(3): 144-149. Available from: http://revistas.pucsp.br/index.php/RFCMS/article/ view/25868

5.Olino L, Gonçalves A C, Strada JKR, Vieira LB, Machado MLP, Molina KL et al . Comunicação efetiva para a segurança do paciente: nota de transferência e Modified Early Warning Score. Rev. Gaúcha Enferm. [Internet]. 2019 [cited 2019 Jan 05] ; 40: e20180341. Available from: http:/www.scielo.br/pdf/rgenf/ v40nspe/1983-1447-rgenf-40-spe-e20180341.pdf

6. Kahl ERPY, Brião RC da Costa, Costa LM, Silveira LR, Moraes MAP. Cenário ambulatorial de pacientes com sítio cirúrgico infectado após intervenção cardíaca. Rev. Gaúcha Enferm [Internet]. 2019 [cited 2019 Dec 02] ; 40: e20180200. Available from: http://www.scielo.br/scielo.php?script=sci_arttext\&pid=S1983-14472019000100422\&lng=en

7. Reis MMR, Lima EFA, Casagrande RI, Fioresi M, Leite FMC, Primo CC. Perfil epidemiológico de pacientes submetidos à cirurgia cardíaca. Rev enferm UFPE on line [internet].2019 [cited 2019 jul 13];13(4):1015-22. Available from: https://periodicos.ufpe.br/ revistas/revistaenfermagem/article/view/238020/31797

8. Dessotte CAM, Figueiredo ML, Rodrigues HF, Furuya RK, Rossi LA, Dantas RAS. Classificação dos pacientes segundo o risco de complicações e mortalidade após cirurgias cardíacas eletivas. Rev Elet. Enf. [Internet]. 2016[cited 2019 apr 8];18:e1140. Available from:: https://revistas.ufg.br/fen/article/view/37736/20966

9. Silva LDC, Melo MVP, Rolim ILTP, Dias RS. Intervenções de enfermagem em pacientes da unidade de terapia intensiva cardiológica de um hospital universitário submetidos à cirurgia de revascularização do miocárdio. JMPH [Internet]. 2018[cited 2019 oct 12]; 9 :e12.Available from: http://www.jmphc.com.br/jmphc/ article/view/510/735

10. Silveira CR, Santos MBK, Moraes MAP, Souza EN. Desfechos clínicos de pacientes submetidos à cirurgia cardíaca em um hospital do noroeste do rio grande do sul. Rev Enferm UFSM [internet].2016 Já[cited 2019 Set 07];6(1):102-111. Available from: https://periodicos.ufsm.br/reufsm/article/view/16467/pdf

11. Orem DE. Nursing: Concepts of practice (6th ed.). St. Louis: Mosby. Padrões de Acreditação da Joint Commission International para Hospitais, $4^{\mathrm{a}}$ ediç̧ão, editado por: Consórcio Brasileiro de Acreditação de Sistemas e Serviços de Saúde - Rio de Janeiro: CBA, 2011.

12. WiegandB. B., \& MeirellesJ. M. L. Desospitalização e cuidados paliativos domiciliares no Sistema Único de Saúde brasileiro. Revista Brasileira De Bioética,[internet]. 2019[cited Nov 11];14:edsup .Available from: https://periodicos.unb.br/index.php/rbb/article/view/24720
13. Robinson CC, Rosa RG, Kochhann R, Schneider D, Sganzerla D, Dietrich C, et al. Qualidade de vida pós-unidades de terapia intensiva: protocolo de estudo de coorte multicêntrico para avaliação de desfechos em longo prazo em sobreviventes de internação em unidades de terapia intensiva brasileiras. Rev Bras Ter intensiva[internet].2018[cited $201911 \mathrm{dec}] ; 30(4): 405-413$. Available from:: http://www.scielo.br/pdf/rbti/v30n4/0103507X-rbti-20180063.pdf

14. Mesquita ET, Cruz LN, Mariano BM, Jorge AJL. Síndrome Pós-Hospitalização: Um Novo Desafio na Prática Cardiovascular. Arq. Bras. Cardiol. [Internet]. 2015 Nov [cited 2019 Nov 05] ; 105 ( 5 ): 540-544. Available from: http://dx.doi.org/10.5935/ abc.20150141.

15. Araújo HVS, Figueirêdo TR, Costa CRB, Silveira MMBM, Belo RMO, Bezerra SMMS. Qualidade de vida de pacientes submetidos à cirurgia de revascularização do miocárdio. Rev Bras Enferm Internet [internet].2017[cited 2019 oct 16]; 70 (2): 27381. Available from: http://www.scielo.br/pdf/reben/v70n2/ pt_0034-7167-reben-70-02-0257.pdf

16. Pissolato JS, Fleck CS, M. SC. Mobilização precoce na unidade de terapia intensiva adulta[Internet].2018 [cited 2019 Nov 13];19(3):377-384. Available from: https://pesquisa.bvsalud. org/portal/resource/pt/biblio-947832

17. Rocha RSB, Gomes TCO, PINTO JM, Oliveira LS, Moreno MA. Cirurgia cardíaca e complicações: uma breve revisão sobre os efeitos da mobilização precoce no paciente crítico. Revista CPAQV - Centro de Pesquisas Avançadas em Qualidade de Vida [Internet].2017 [cited 2019 apr 10];9(2):2-4. Available from: http://www.cpaqv.org/revista/CPAQV/ojs-2.3.7/index.php?journal $=$ CPAQV\&page $=$ article\&op=view\&path $\% 5 B \% 5 \mathrm{D}=203$

18. Oliveira J FP, Bonomo LMM, Brito FA, Costa RM, Tótola CR, Gomes FLT. Assistência fisioterapêutica a um paciente submetido à cirurgia de revascularização do miocárdio com evolução de mediastinite como complicação pós-operatória. Fisioterapia Brasil[internet] 2019[cited 2019 oct 12]; 20 (4): p. 546-553. Available from: http://www.portalatlanticaeditora.com.br/index. php/fisioterapiabrasil/article/view/2652/pdf

19. Monteleone S, Dalla TE, Emiliani V, Ricotti S, Bruggi M, Conte $T$, et al. Recovery of deambulation after cardio-thoracic surgery: a single center experience. Eur J Phys Rehabil Med.[internet]2015[cited 2019 apr 09] ;51(6):763-71. Available from: https://www.minervamedica.it/en/journals/europa-medicophysica/article.php?cod=R33Y2015N06A0763

20. Silva LLT, Mata LRF, Silva AF, Daniel JC, Andrade AFL, Santos ETM. Cuidados de enfermagem nas complicações no pós-operatório de cirurgia de revascularização do miocárdio. Rev baiana enferm[Internet].2017[cited 2019 nov 18]; 31(3):e20181. Available from: https:/rigs.ufba.br/index.php/enfermagem/article/ view/20181/15030

21. Santos VN, Byk J. Assistência espiritual/religiosa a pacientes hospitalizados: revisão narrativa. Psic., Saúde \& Doenças [Internet]. 2019 [cited 2019 Dec 05] ; 20( 2 ): 348-357. Available from: http://dx.doi.org/10.15309/19psd200206 